\title{
Prevalência do consumo de risco de álcool no idoso: estudo numa unidade dos cuidados primários da região de Braga
}

Albino Martins, ${ }^{1}$ Joana Parente, ${ }^{1}$ Joana Araújo, ${ }^{2}$ Maria José Menezes $^{3}$

\section{RESUMO}

Introdução: O consumo de álcool está associado a riscos e benefícios dependendo da quantidade ingerida. Perceber os padrões de consumo de uma população torna-se fundamental na prevenção dos riscos associados ao álcool. Os idosos, pelas suas particularidades, representam um grupo de interesse maior, que atualmente está subestudado nesta área. Considerando o papel central dos médicos de família na avaliação global do utente, a sua ação preventiva é fulcral.

Objetivos: Estimar a prevalência do consumo de risco do álcool em idosos numa unidade de cuidados primários e caracterizar a população em estudo.

Métodos: Estudo transversal de uma população de 1.225 indivíduos com 65 ou mais anos de idade. Definiu-se uma amostra aleatória representativa constituída por 210 idosos. A recolha dos dados foi efetuada com recurso à ficha individual do utente. Por consumo de risco definiu-se aquele superior ou igual a 14 unidades padrão de etanol (168g) por semana.

Resultados: Da amostra de 210 idosos, com idade média de 73,7士7,7 anos, 57,6\% eram mulheres. A prevalência encontrada para o consumo de álcool foi de 63\% (IC95\%: 56-69) e de consumo de risco de 32,9\% (IC95\%: 26-39) - nos homens 56,2\% (IC95\%: 49-62) e nas mulheres 15,7\% (IC95\%: 10-20), sendo 36,7\% (IC95\%: 30-41) da amostra abstinente. Verificou-se associação significativa entre o género masculino e o consumo de risco do álcool. A idade e a escolaridade não apresentaram associação com o consumo de risco.

Discussão: No idoso, o consumo excessivo de álcool pode ter consequências particularmente gravosas. Não obstante, verifica-se uma escassez de estudos que avaliem o padrão de consumo de álcool nesta população. Observou-se que 63,3\% dos idosos consome álcool, com predomínio do género masculino, o que não difere dos dados nacionais. De realçar, porém, que a prevalência do consumo de risco, que inclui um terço da amostra, é superior à encontrada noutros estudos similares.

Conclusão: O consumo de risco do álcool é prevalente no idoso, pelo que o médico de família deve estar consciente deste problema.

Palavras-chave: Etanol; Idoso.

\section{INTRODUÇÃO}

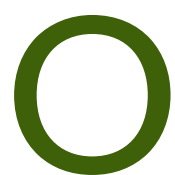

álcool é uma substância cujo consumo faz parte dos hábitos alimentares da sociedade em geral, integrado tanto na vida quotidiana e familiar como em eventos sociais e cerimónias religiosas. ${ }^{1-2} \mathrm{O}$ seu consumo moderado tem sido associado a benefícios para a saúde a médio e longo pra-

${ }^{1}$ Médicos Internos de Medicina Geral e Familiar. USF de S. Lourenço, Aces do Cávado I - Braga

${ }^{2}$ Médica de Medicina Geral e Familiar. USF de S. Lourenço, Aces do Cávado I - Braga ${ }^{3}$ Médica de Medicina Geral e Familiar. USF de S. Lourenço, Aces do Cávado I - Braga zo. ${ }^{2-3}$ Todavia, o uso de álcool em quantidades desaconselhadas pode estar relacionado com inúmeras causas de morbimortalidade, que incluem desde acidentes rodoviários a doenças hepáticas, cardiovasculares, psiquiátricas, pulmonares, imunológicas, ósseas e musculares, gastrointestinais, entre muitas outras. ${ }^{2,4}$ Segundo a Organização Mundial da Saúde (OMS), o consumo de álcool figura entre os dez principais fatores de risco de doenças. ${ }^{1}$

Apesar de o consumo excessivo de álcool estar frequentemente associado a jovens adultos, esta questão é multigeracional. ${ }^{5}$ 
Atualmente, os idosos, definidos como aqueles com idade igual ou superior a 65 anos, estão subestudados no que diz respeito aos padrões de consumo de álcool, existindo poucos dados exclusivos relativos a este grupo etário. ${ }^{6}$ No idoso, a quantidade máxima diária recomendada pelas normativas internacionais é de uma bebida padrão (BP) (cerca de 12g de álcool), independentemente do género. ${ }^{6} \mathrm{~A}$ BP é uma unidade de medida que avalia o volume de álcool numa bebida alcoólica, o que permite o cálculo da ingestão diária ou semanal de álcool. ${ }^{7}$

A falta de estudos sobre este tema tem especial interesse na medida em que, durante o século XX, o número de idosos europeus triplicou e a esperança média de vida aumentou para mais do dobro, estimando-se que em 2028 mais de um quarto da população europeia terá 65 ou mais anos. ${ }^{5}$

Considerando o envelhecimento populacional, o consumo de álcool nos idosos é um assunto premente que merece mais atenção, bem como o diagnóstico e tratamento dos problemas relacionados com o álcool neste grupo etário. Além disso, os idosos, pelas comorbilidades que geralmente lhes estão associadas e pelo uso comum de medicamentos, constituem um grupo de maior risco.

O abuso do álcool é um conceito global que inclui desde o consumo de risco até à dependência. Por consumo de risco entende-se um padrão de consumo que poderá levar a consequências físicas, mentais ou sociais deletérias para o próprio ou terceiros. ${ }^{7-8}$ De acordo com a recomendação da entidade norte-americana National Institute on Alcohol Abuse and Alcoholism, é considerada uma ingestão de álcool diária de baixo risco no idoso aquela igual ou inferior a uma BP. ${ }^{6}$ Em concordância com esta recomendação, o consumo de risco do álcool no idoso pode ser definido como aquele igual ou superior a duas BP por dia. Esta definição não é, porém, consensual entre diferentes autores e diferentes países. ${ }^{8}$

Os profissionais dos cuidados de saúde primários (CSP) usufruem de uma posição privilegiada para a deteção e intervenção precoces em padrões de consumo que vão além do moderado, tendo em conta a avaliação multicêntrica do utente e o seu seguimento longitudinal. De referir ainda o respeito e confiança depositada, por parte do utente, no seu médico de família, o que certamente aumentará o sucesso da intervenção educacional. ${ }^{1,3,5}$

Neste contexto, e dado o reduzido número de estudos que abordam este tema na população geriátrica, fo- ram objetivos do presente trabalho estimar a prevalência do consumo de risco de álcool numa população de utentes idosos no contexto dos CSP e caracterizar a população em estudo em relação ao consumo crónico de fármacos e variáveis sociodemográficas.

\section{MÉTODOS}

Estudo observacional transversal de uma população de utentes com idade superior ou igual a 65 anos de idade, inscritos até 31 de dezembro de 2013 numa USF da região do Minho. De um total de 1.225 idosos definiu-se amostra aleatória de 210 indivíduos, de acordo com a proporção estimada de consumo de risco de $10 \%$, obtida na literatura internacional, e um erro amostral de $5 \% .{ }^{9}$ As variáveis estudadas foram a idade, o género, a escolaridade, o consumo de álcool e a polimedicação. Na obtenção dos dados foram utilizados os registos informatizados do Sistema de Apoio ao Médico (Ficha Individual do Utente). Definiu-se consumo de risco de álcool como um valor médio de ingestão semanal superior ou igual a 168 gramas (14 BP), em ambos os géneros. ${ }^{7-8}$ Definiu-se por polimedicação o uso simultâneo de cinco ou mais fármacos de uso crónico. Excluíram-se os utentes sem informação sobre o consumo de etanol nos vinte e quatro meses precedentes à recolha dos dados, sendo substituídos de acordo com a ordem de aleatorização previamente estabelecida. A análise estatística foi efetuada com recurso ao Statistical Package for the Social Sciences, versão 21 (IBM $\left.{ }^{\circledR}\right)$, com um nível de significância estatística de $5 \%(p<0,05)$. Foi utilizado o teste de Qui-quadrado para comparação entre variáveis qualitativas. As variáveis contínuas foram comparadas com recurso ao teste $t \mathrm{e}$ análise ANOVA. O coeficiente de correlação de Pearson foi utilizado para avaliar o grau da correlação entre variáveis métricas.

O estudo foi submetido a parecer da Comissão de Ética para a Saúde da Administração Regional de Saúde do Norte, de que resultou a aprovação em reunião de 7 de abril de 2015.

\section{RESULTADOS}

Estudaram-se 210 utentes, $57,6 \%$ mulheres, com idade média de 75,3 $\pm 7,7$ anos, com valor mínimo de 65 e máximo de 95 anos. Da amostra inicial foram excluídos 27 idosos (12,9\% do total) por ausência de informação sobre o consumo de etanol. Entre os 65 e os 75 anos de idade encontram-se $49,5 \%$ dos idosos em estudo. Em 
média, obtiveram-se 2,4 $\pm 1,3$ registos do consumo de álcool por idoso. O consumo médio, em gramas, foi de $99,5 \pm 104 \mathrm{~g}$ de álcool por semana. A prevalência encontrada para uso de álcool foi de cerca de $63 \%$ (IC95\%: 5669). A prevalência encontrada para consumo de risco do álcool foi de 32,9\% (IC95\%: 26-39) - 56,2\% (IC95\%: 4962) nos homens e 15,7\% (IC95\%: 10-20) nas mulheres. Contudo, 36,7\% (IC95\%: 30-41) da população revelou-se abstinente para o consumo de álcool. Verificou-se existir uma associação significativa entre género masculino e consumo de risco do álcool (56,2\% no género masculino comparativamente com $15,7 \%$ no género feminino; $p<0,01)$. Por outro lado, no género feminino encontrou-se uma associação significativa com a abstinência do álcool $(p<0,01)$ sendo que, dos idosos abstinentes, $72,7 \%$ eram mulheres. Não se verificou correlação entre a progressão da idade e o consumo de álcool em gramas $(p=0,08)$. Quando avaliados escalões etários com intervalos de 10 anos, também não se verificaram diferenças no consumo médio de álcool $(p=0,23)$. Analisando os idosos com ou sem consumo de risco do álcool verifica-se que aqueles com consumo de risco são, em média, mais jovens (73,8 anos $v s .76,1$ anos; $p=0,04$ ). Quando comparados os idosos com seis ou mais anos de escolaridade ( $11,2 \%$ do total) com aqueles com menor escolaridade não se verificou diferença na média do consumo de álcool $(75,8 \mathrm{~g} v$ s. $113,5 \mathrm{~g} ; p=0,11)$ ou associação com consumo de risco $(22,7 \%$ vs. $37,6 \%$; $p=0,12$ ). Nos idosos com consumo de risco verificou-se que $50,7 \%$ encontra-se polimedicado ( $56 \%$ de polimedicação nos idosos sem consumo de risco).

\section{DISCUSSÃO}

A prevenção das consequências relacionadas com o consumo de álcool apenas é possível com o conhecimento dos padrões de consumo na população. ${ }^{2}$ No idoso, o consumo excessivo de álcool pode ter consequências particularmente graves, dada a maior suscetibilidade aos seus efeitos e associação com risco acrescido de disfunção cognitiva e demência. ${ }^{8,10}$ Neste ponto realça-se a importância da intervenção dos cuidados de saúde primários na avaliação e acompanhamento do padrão de consumo e das particularidades específicas desta população. ${ }^{10}$

A prevalência encontrada para uso de álcool foi de cerca de 63\% (IC95\%: 56-69). Os estudos nacionais es- timam na população idosa uma prevalência para uso de bebidas alcoólicas no último ano de $52 \%$, com predomínio do género masculino. ${ }^{10}$ Esta diferença pode ser explicada por fatores regionais, dada a prevalência de consumo de álcool na região norte ser superior em todos os grupos etários comparativamente com o restante território continental. ${ }^{11}$

No que concerne ao consumo de risco de álcool na população estudada, a prevalência estimada foi de $32,9 \%$. O abuso de álcool é um conceito global que inclui desde o consumo de risco até à dependência. São escassos os estudos que avaliam diretamente o consumo de risco do álcool no idoso. Nos Estados Unidos da América, as estatísticas mostram que cerca de $10 \%$ da população idosa abusa do álcool ou consome-o de forma problemática. ${ }^{5}$ Connell e colaboradores estimaram que a prevalência de consumo excessivo nos idosos possa rondar os $17 \%$ no homem e $7 \%$ na mulher. ${ }^{12}$ Esta assimetria de género foi também observada na amostra em estudo (52,2\% vs. 15,75\%), havendo uma associação estatisticamente significativa entre género masculino e consumo de risco. Esta associação foi previamente demonstrada noutras populações idosas e parece estar intimamente ligada com fatores socioculturais. ${ }^{13-14}$

Não foi feita estratificação da amostra por classe etária ou nível educacional. Contudo, a análise estatística destas variáveis não mostrou associação com consumo de risco. Porém, níveis educacionais baixos estão geralmente associados a maior prevalência de distúrbios relacionados com o álcool. ${ }^{15}$

Geralmente os idosos consomem menos álcool e têm menos problemas relacionados com o seu consumo que os indivíduos mais jovens. ${ }^{6}$ Curiosamente, neste trabalho, esta tendência é também observada quando comparado o consumo de risco entre diferentes classes etárias acima dos 65 anos.

Naqueles idosos com consumo de risco, a maioria encontrava-se polimedicada. O uso conjunto do álcool e de vários medicamentos aumenta o risco de toxicidade, pelo que deve ser dada particular atenção a esta circunstância.

De realçar que o consumo de risco do álcool foi prevalente, abrangendo um terço da população estudada. Vários estudos têm demonstrado uma prevalência crescente de problemas relacionados com o álcool no idoso, apesar da menor atenção pública dada à questão. 
Embora por vezes difícil, a correta quantificação do consumo e avaliação dos problemas de saúde associados com o álcool são fulcrais para evitar e reduzir esta epidemia silenciosa. ${ }^{6,15}$

Este estudo de caráter local teve por propósito o diagnóstico do consumo de risco de álcool na população idosa e abriu a possibilidade para uma intervenção mais informada neste contexto. É necessária uma avaliação futura mais pormenorizada deste problema na população idosa, idealmente com recurso a estudos de base multicêntrica e mais alargada. A reduzida dimensão amostral, a possível existência de variáveis de confundimento, a fiabilidade dos dados induzida pelo consumo autorreportado e o viés de seleção condicionado pela exclusão dos utentes sem informação sobre consumo do álcool limitam a generalização dos resultados a outras populações. Conclui-se que o consumo de risco do álcool no idoso é prevalente. Deste modo, os autores consideram necessária a promoção de práticas de prevenção do consumo excessivo do álcool nos CSP, em concordância com o preconizado pelo programa de intervenção governamental. ${ }^{16}$

\section{REFERÊNCIAS BIBLIOGRÁFICAS}

1. Varela MF. Prevalência do consumo do álcool em dois centros de saúde da Praia e num centro numa zona rural, Picos - Ilha de Santiago [Dissertation]. Lisboa: Faculdade de Ciências Médicas, Universidade Nova de Lisboa; 2013. Available from: http://hdl.handle.net/10362/8861

2. World Health Organization. International guide for monitoring alcohol consumption and related harm [Internet]. Geneva:WHO; 2000. Available from: http://apps.who.int/iris/bitstream/10665/66529/1/ WHO_MSD_MSB_00.4.pdf

3. National Institute on Alcohol Abuse and Alcoholism. NIAAA releases physicians' guide [Internet]. Washington, DC: NIAAA; 1995. Available from: https://www.niaaa.nih.gov/news-events/news-releases/niaaa-releases-physicians-guide

4. Andersen P, Baumberg B. O álcool na Europa [Internet]. Lisboa: Sociedade Anti-Alcoólica Portuguesa, Instituto S. João de Deus; 2006. Available from: http://btg.ias.org.uk/pdfs/alcohol-in-europe/country-translations/portugal.pdf

5. Observatório Europeu da Droga e da Toxicodependência. Drogas em destaque. Lisboa: OEDT; 2008.

6. National Institute on Alcohol Abuse and Alcoholism. Washington, DC: NIAAA Page [Internet]; 1970 Dec 31 [updated 2016]. Available from: http://www.niaaa.nih.gov/

7. Centers for Disease Control and Prevention. Alcohol use and your health: fact sheets [Internet]. Atlanta: CDC; 2014 [updated 2016 Feb 29]. Available from: www.cdc.gov/alcohol/fact-sheets/alcohol-use.htm

8. International Center for Alcohol Policies. International drinking guidelines [Internet]. Washington: ICAP; 2003. Available from:
http://www.icap.org/portals/0/download/all_pdfs/ICAP_Reports_English/report14.pdf

9. Observatório Europeu da Droga e da Toxicodependência. Observatório Europeu da Droga e da Toxicodependência: quem somos, o que fazemos [Internet]. Lisboa: OEDT; 2009. Available from: http://bookshop.europa.eu/pt/observat-rio-europeu-da-droga-e-da-toxicodependncia-pbTD3008565/?CatalogCategoryID=9lUKABst9YMAAAEjTYcY4e5K

10. Serviço de Intervenção nos Comportamentos Aditivos e nas Toxicodependências. Plano nacional para a redução dos comportamentos aditivos e das dependências, 2013-2020 [Internet]. Lisboa: Ministério da Saúde; 2013. ISBN 9789729345920. Available from: http://www.sicad.pt/BK/Institucional/Coordenacao/Documents/Planos/SICAD_Plano_Nacional_Reducao_CAD_2013-2020.pdf

11. Felício $M M, M a c h a d o ~ V$, Teixeira $C$. Perfil de saúde da região Norte [Internet]. Porto: Administração Regional de Saúde do Norte; 2009. Available from: http://portal.arsnorte.min-saude.pt/portal/page/portal/ ARSNorte/Conte\%C3\%BAdos/Sa\%C3\%BAde\%20P\%C3\%BAblica\%20Conteudos/Perfil_RN_2009.pdf

12. O'Connell H, Chin AV, Cunningham C, Lawlor B. Alcohol use disorders in elderly people: redefining an age old problem in old age. BMJ. 2013; 327(7416):664-7.

13. Saunders PA, Copeland JR, Dewey ME, Davidson IA, McWilliam C, Sharma $V$, et al. Heavy drinking as a risk factor for depression and dementia in elderly men: findings from the Liverpool longitudinal community study. Br J Psychiatry. 1991;159:213-6.

14. Iliffe S, Haines A, Booroff A, Goldenberg E, Morgan P, Gallivan S. Alcohol consumption by elderly people: a general practice survey. Age Ageing. 1991;20(2):120-3.

15. Centro de Informações sobre Saúde e Álcool. São Paulo, BR: CISA Page [Internet]; 2003 [updated 2016]. Available from: http://www.cisa.org.br/ index.php

16. Ministério da Saúde. Relatório do Grupo de Trabalho de elaboração do Programa de deteção precoce e intervenções breves dirigido ao consumo excessivo de álcool e tabaco nos Cuidados de Saúde Primários: relatório do subgrupo (álcool) [Internet]. Lisboa: Ministério da Saúde; 2015. Available from: http://www2.portaldasaude.pt/NR/rdonlyres/ C17E8534-6D1A-4FE9-9371-43782CF2C590/0/Relat\%C3\%B3rioGT _Dete\%C3\%A7\%C3\%A3oeinterven\%C3\%A7\%C3\%B5esbreves_CSP_ alcool_final3.pdf

\section{CONFLITOS DE INTERESSE}

Os autores declaram não ter conflitos de interesse.

\section{COMISSÃO DE ÉTICA}

Estudo realizado após parecer favorável da Comissão de Ética da ARS Norte.

\section{ENDEREÇO PARA CORRESPONDÊNCIA}

Albino Martins

Trav. S. André, n 186, 4795-152 Vila das Aves, Porto

E-mail: albinomartins.uminho@gmail.com

Recebido em 24-07-2015

Aceite para publicação em 02-06-2016 


\section{ABSTRACT}

\section{PREVALENCE OF RISKY ALCOHOL CONSUMPTION IN THE ELDERLY:A STUDY FROM A PRIMARY CARE UNIT \\ IN THE BRAGA REGION}

Introduction: Alcohol consumption is associated with risks and benefits depending on the quantity ingested. It is helpful to understand patterns of alcohol consumption in a population in order to decrease risk. The elderly represent a group of special interest, currently under-studied in this area. Family physicians can play a role in the evaluation and prevention of risky alcohol consumption in the elderly.

Objectives: To assess the prevalence of risky alcohol consumption in the elderly in a primary care unit.

Methods: A cross-sectional study of a sample the clinical records of an 1,225 individuals aged 65 or over was conducted. A representative random sample of 210 elderly patients was selected. Risky consumption was defined as 14 or more standard ethanol units (168g) per week.

Results: In this sample of 210 elderly patients, the mean age was $73.7 \pm 7.7$ years and $57.6 \%$ were women. The prevalence of alcohol consumption was $63 \%(95 \% \mathrm{Cl} 56,69)$ and risky consumption was found in $32.9 \%(95 \% \mathrm{Cl} 26,39)[56.2 \%(95 \% \mathrm{Cl} 49,62)$ in men, $15.7 \%(95 \% \mathrm{Cl} 10,20)$ in women], with $36.7 \%(95 \% \mathrm{Cl} 30,41)$ of the sample who were abstinent. We found a significant association between male gender and risky alcohol consumption. Age and education were not related to risky consumption. Discussion: In the elderly, excessive consumption of alcohol can have serious consequences. There is a lack of studies to assess patterns of alcohol consumption in this population. We found that $63.3 \%$ of the elderly in this population consume alcohol, with a male predominance, similar to findings in national studies. The prevalence of the risky consumption in this population, which includes a third of the sample, is higher than that found in other similar studies.

Conclusion: Alcohol risk consumption is prevalent in the elderly in this population and family physicians should be aware of this problem.

Keywords: Ethanol; Aged. 